\title{
Amino Acid Substitutions Analysis of the Putative Epitopes of Neuraminidase Protein from Influenza A/H1N1 Virus
}

\author{
Amino Acid Substitutions Analysis of the Putative Epitopes of \\ Neuraminidase Protein from Influenza A/H1N1 Virus
}

\author{
Ludmila Alves Dias Souto ${ }^{1}$ \\ Alessandra Rejane Ericsson de Oliveira Xavier² \\ Mauro Aparecido de Sousa Xavier ${ }^{3}$
}

\begin{abstract}
Objective: This study verified whether the neuraminidase protein of Influenza A H1N1 virus sequence has modified from 2009-2017 and its impact on the 2018 Brazilian vaccine. Method: The reference neuraminidase protein sequence from H1N1 Puerto Rico/1934 strain was subjected to three different methods of epitope prediction and the top five from each method were aligned using Clustal omega, resulting in eight putative epitopes. These epitopes were aligned to 7,438 neuraminidase sequences spanning from 2009-2017 and analyzed for specific amino acid substitutions and counted. The resultant neuraminidase protein was aligned against the 2015 and 2018 neuraminidase proteins, from Influenza A H1N1 virus subtypes, used for vaccine production. Result: Twenty-one main substitutions were detected, of which 16/21 (76.2\%) substitutions points remained stable and 1/21 (4.8\%) returned to the original amino acid residue in the viral population from 2009-2017. Additionally, 19\% (4/21) substitutions occurred in Brazil and worldwide in this period, indicating that changes in the neuraminidase viral population profile is time-dependent rather than geographical. Conclusion: The neuraminidase protein containing these amino acid substitutions is more closely related to the neuraminidase protein from influenza A/Michigan/45/2015 than A/California/7/2009, supporting the replacement of this virus subtype in the Brazilian vaccine in 2018.
\end{abstract}

Key words: Bioinformatics; Glycoprotein; Mutation; Neuraminidase; Vaccine.

\footnotetext{
${ }^{1}$ Mestre em Biotecnologia (Unimontes). Analista de Produção (Novo Nordisk Produção Farmacêutica do Brasil) (NNPFB). Minas Gerais. Brasil. $₫$ ludmila.ads@ hotmail.com (iD https://orcid.org/0000-0003-1145-131x

${ }^{2}$ Doutora em Biologia Molecular (UnB). Professora da Universidade Estadual de Montes Claros (Unimontes). Minas Gerais. Brasil. $₫$ Ericsson_aerc@yahoo.com.br. (D) https://orcid.org/0000-0001-8558-4196

${ }^{3}$ Doutor em Biologia Molecular (UnB). Professor da Universidade Estadual de Montes Claros (Unimontes). Minas Gerais. Brasil. $₫$ mauro.xavier@unimontes.br. (iD) https://orcid.org/ 0000-0002-0512-1616
}

\begin{tabular}{c|ccc} 
Recebido em & $\begin{array}{c}\text { Correções } \\
\text { solicitadas em } \\
17 / 08 / 2020\end{array}$ & Aceito em & Publicado em \\
$06 / 06 / 2020$ & $18 / 08 / 2020$ & $07 / 09 / 2020$
\end{tabular}




\section{INTRODUCTION}

The influenza virus is the highest cases of morbidity and mortality every year, causing a significant impact on global public health care. ${ }^{1-5}$ Approximately $3-20 \%$ of the world's population is infected each year by the influenza virus and its genome mutation rate is estimated to be $2.3 \times 10^{-5}$ mutations per nucleotide by infected-cell, resulting in millions of infected cells during the disease. ${ }^{6,7}$ Among the mutations acquired by the influenza virus, termed antigenic drift, the hemagglutinin (HA) and neuraminidase (NA) glycoproteins are important because they bind to the cell surface. ${ }^{1,7}$ Although HA is the primary target for neutralizing antibodies, antibodies against NA can also reduce the occurrence and severity of the disease, and possibly prevent infection when present in high viral titers. ${ }^{7,8}$

The glycoprotein NA of the virus H1N1 surface recognize the sialic acid from the surface of the host cell and break down the previous binding between the HA protein and its receptor in the infected cell, allowing that virus particle to enter the host cell. ${ }^{9,10}$ Additionally, the sialidase activity of the NA protein is critical for releasing new virus particles from the cell surface of infected cells. ${ }^{11,12}$ NA may also play a role early in infection and may facilitate the entry of the virus and/or intensifying the late trafficking of the endosome and/or lysosome. .,12,13 $^{2}$ These characteristics have made NA a focus of research, becoming a common target for antibodies and consequently for drugs and vaccines development. Amino acid substitution in the NA protein may lead to loss of sensibility to inhibitors of viral neuraminidase activity. ${ }^{11,14,15}$

Studies of viral mutations in vivo or accompanied by predictive models using computer programs have been described in the literature, for example, those reporting mutations for NA gene or amino acid substitution in influenza A (H1N1) pdm09 virus, which conferred resistance to the inhibitors drugs of viral surface glycoproteins. . $^{2,10,16,17}$

The World Health Organization (WHO) convenes technical consultations twice a year, in February/March and September, to recommend virus strains to be included in the vaccines used for the northern and southern hemispheres, respectively, for the next influenza season. A recommendation changing influenza A (H1N1) strain was made in September 2017 for vaccines to be used for the southern hemisphere influenza season $2018 .{ }^{18}$ Due to this recommendation, the vaccine used in Brazil to prevent the influenza A (H1N1) virus infection 
from 2009-2017 containing subtype A/California/7/2009 virus was replaced by subtype A/Michigan/45/2015 to be marketed in $2018 .{ }^{19}$

In the view of above, the objective of this study was to analyze NA protein sequences and amino acids substitutions through bioinformatics programs and compare amino acid sequences from 2009-2017, and to collect those substitutions data to evolutionary predictions on NA protein of the influenza A (H1N1) virus. In addition, it was verified whether the viral subtype containing NA in the vaccine used in Brazil was the most appropriate and/or whether it needs to be replaced with another viral subtype.

\section{METHOD}

The NA protein sequences in the influenza A (H1N1) virus were obtained from bioproject data bank available at https://www.ncbi.nlm.nih.gov/bioproject/PRJNA37813. In December 2017, there were 68,274 protein sequences in the databank for the eight main protein components of the viral particle including NA protein (entry date: December 10, 2017). The NA protein (469 aa) sequences were selected using filter to sequence the length from 465 to 475, and resulting in 7,438 sequences. All NA sequences were downloaded for further analysis.

The NA protein sequence of Influenza A virus subtype A/Puerto Rico/8/1934 (H1N1), GenBank: ABO21711.1, was used as a reference sequence because it was the first influenza A $\mathrm{H} 1 \mathrm{~N} 1$ virus described in the pandemic disease. The epitope prediction in this protein was done by three different methods, i.e., (1) Bepipred linear epitope prediction (http://tools.immuneepitope.org/bcell/), (2) Kolaskar \& Tongaonkar Antigenicity methods (http://tools.immuneepitope.org/bcell/) and also by (3) CTLPred antigenic method (http://crdd.osdd.net/raghava/ctlpred/). From the Bepipred and Kolaskar \& Tongaonkar methods, the top five peptides were selected based on the highest aminoacid residue score that belong to the corresponding epitope sequence. However, the CLTPred method was programmed to show the top five putative epitopes based on nine amino acid residues length from the protein sequence. Afterwards, the 15 putative epitopes obtained form the three methods were aligned with the reference protein using the Clustal omega software (www.ebi.ac.uk/Tools/services/rest/clustalo/), and the complete sequences contaning the putative amino acid residues from the predicted and eight selected epitopes were obtained.

The predicted epitopes (8) were aligned with the 7,438 neuraminidase sequences using the Clustal omega software (www.ebi.ac.uk/Tools/services/rest/clustalo/), and the amino acids substitutions in each epitope were noticed manually. Each specific amino acid substitution was 
counted by year and its frequency calculated in percentage. Besides, the selected epitopes were used to align with 138 NA sequences of influenza A (H1N1) from Brazil (Obtained from IRD - Influenza Research Database) and the frequency of each amino acid substitution was calculated.

The selected epitopes were modified to contain the replaced amino acid residues from the virus population circulating in 2017 and aligned against NA sequence from influenza H1N1 virus subtypes A/California/07/2009 and A/Michigan/45/2015. The influenza A/California/07/2009 neuraminidase (Uniprot: C3W6G3) was included in the Brazilian vaccine until 2017, and it was replaced by the virus subtype A/Michigan/45/2015 (Uniprot: A0A0X9QTS2). The new subtype virus will then be part of the Brazilian vaccine against influenza to be utilized by the Brazilian public health system for immunization from 2018, as recommended by World Health Organization, ${ }^{18}$ and described in the resolution number 2,696 from October 2017 by the Brazilian Health Minister. ${ }^{19}$

\section{RESULTS}

The reference sequence of influenza A (H1N1) neuraminidase used for epitopes prediction was subtype A/PuertoRico/8/1934 (GenBank: ABO21711.1). The linear prediction Bepipred and the Kolaskar \& Tongaonkar methods suggested 14 and 16 putative epitopes (TAB. 1), respectively, considering only peptides containing more than seven amino acid residues length. However, the CLTPred showed the five epitopes sequences as requested for 9 nucleotides length (TAB. 1).

Table 1 - Putative epitopes from neuraminidase of influenza A/Puerto Rico/8/1934 (H1N1) virus predicted by Bebipred linear (BL), Kolaskar \& Tongaonkar (K\&T), and CTL Pred (CTL) methods. The top five epitopes were selected by highest individual amino acid residue score are in italic for each method.

\begin{tabular}{cccccc}
\hline Number \# & Method & Start & End & Peptide sequence & Length \\
\hline 1 & BL & 38 & 48 & IQTGSQNHTGI & 11 \\
2 & BL & 84 & 96 & IYSKDNSIRIGSK & 13 \\
3 & BL & 125 & 140 & LNDKHSSGTVKDRSPY & 16 \\
4 & BL & 147 & 159 & $P V G E A P S P Y N S R F$ & 13 \\
5 & BL & 180 & 188 & ISGPDNGAV & 9 \\
6 & BL & 228 & 236 & TDGPSDGLA & 9 \\
7 & BL & 253 & 271 & ELNAPNSHYEECSCYPDTG & 19 \\
8 & BL & 281 & 290 & WHGSNRPWVS & 10 \\
9 & BL & 306 & 333 & VFGDNPRPEDGGSCGPVYVDGANGKG & 28
\end{tabular}




\begin{tabular}{|c|c|c|c|c|c|}
\hline 10 & BL & 347 & 353 & TKSHSSR & 7 \\
\hline 11 & $\mathrm{BL}$ & 360 & 373 & WDPNGWTETDSKFS & 14 \\
\hline 12 & BL & 383 & 398 & DWSGYSGSFVQHPELT & 16 \\
\hline 13 & $\mathrm{BL}$ & 414 & 423 & GRPKEKTIWT & 10 \\
\hline 14 & $\mathrm{BL}$ & 433 & 451 & VNSDTVDWSWPDGAELPFS & 19 \\
\hline 1 & $\mathrm{~K} \& \mathrm{~T}$ & 8 & 27 & ITIGSICLVVGLISLILQIG & 20 \\
\hline 2 & $\mathrm{~K} \& \mathrm{~T}$ & 31 & 37 & SIWISHS & 7 \\
\hline 3 & $\mathrm{~K} \& \mathrm{~T}$ & 74 & 80 & SSLCPIR & 7 \\
\hline 4 & $\mathrm{~K} \& \mathrm{~T}$ & 98 & 117 & DVFVIREPFISCSHLECRTF & 20 \\
\hline 5 & $\mathrm{~K} \& \mathrm{~T}$ & 140 & 151 & YRALMSCPVGEA & 12 \\
\hline 6 & $\mathrm{~K} \& \mathrm{~T}$ & 162 & 169 & VAWSASAC & 8 \\
\hline 7 & $\mathrm{~K} \& \mathrm{~T}$ & 187 & 194 & AVAVLKYN & 8 \\
\hline 8 & $\mathrm{~K} \& \mathrm{~T}$ & 215 & 225 & ECACVNGSCFT & 11 \\
\hline 9 & $\mathrm{~K} \& \mathrm{~T}$ & 262 & 270 & EECSCYPDT & 9 \\
\hline 10 & $\mathrm{~K} \& \mathrm{~T}$ & 272 & 279 & $K V M C V C R D$ & 8 \\
\hline 11 & $\mathrm{~K} \& \mathrm{~T}$ & 298 & 306 & $Q I G Y I C S G V$ & 9 \\
\hline 12 & $\mathrm{~K} \& \mathrm{~T}$ & 320 & 326 & $C G P V Y V D$ & 7 \\
\hline 13 & $\mathrm{~K} \& \mathrm{~T}$ & 375 & 381 & RQDVVAM & 7 \\
\hline 14 & $\mathrm{~K} \& \mathrm{~T}$ & 389 & 395 & GSFVQHP & 7 \\
\hline 15 & $\mathrm{~K} \& \mathrm{~T}$ & 403 & 412 & MRPCFWVELI & 10 \\
\hline 16 & $\mathrm{~K} \& \mathrm{~T}$ & 427 & 436 & SISFCGVNSD & 10 \\
\hline 1 & CTL & 318 & 326 & GSCGPVYVD & 9 \\
\hline 2 & CTL & 157 & 165 & SRFESVAWS & 9 \\
\hline 3 & CTL & 188 & 196 & VAVLKYNGI & 9 \\
\hline 4 & CTL & 193 & 201 & YNGIITETI & 9 \\
\hline 5 & CTL & 417 & 425 & KEKTIWTSA & 9 \\
\hline
\end{tabular}

Source: Authors' data

The presumed 15 epitopes obtained from all three methods (top five from each method) were schematized as shown in Figure 1A and Figure 1B. The 15 presumed and selected epitopes were aligned with the reference protein using the Clustal omega program (https://www.ebi.ac.uk/Tools/msa/clustalo/), resulting in 8 putative epitopes (Figure 1C) and they were used for searching amino acid substitutions against 7,438 neuraminidase proteins sequences. The Kolaskar \&Tongaonkar method suggested epitopes 1, 3, 5, 7, and 8, while the Bepipred method recommended the epitopes 2, 3, 4, 6, and 7, as well as, the CLTPred method proposed the epitopes $2,3,6$, and 8 . Only putative epitope 3 was suggested by all used methods. 
A

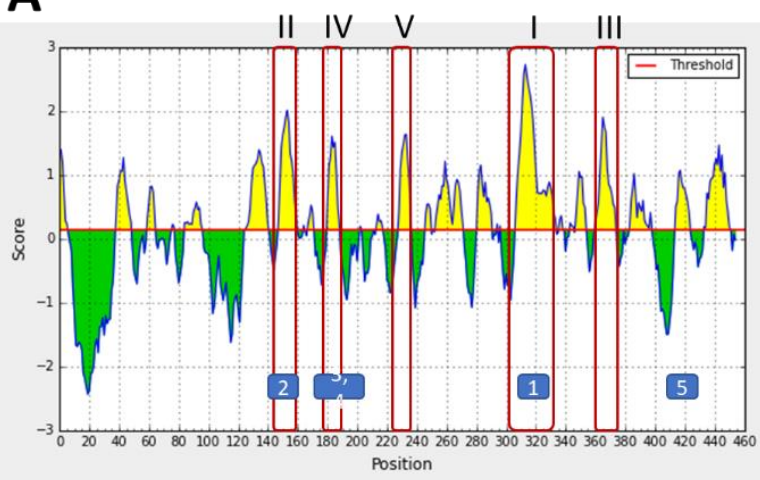

B

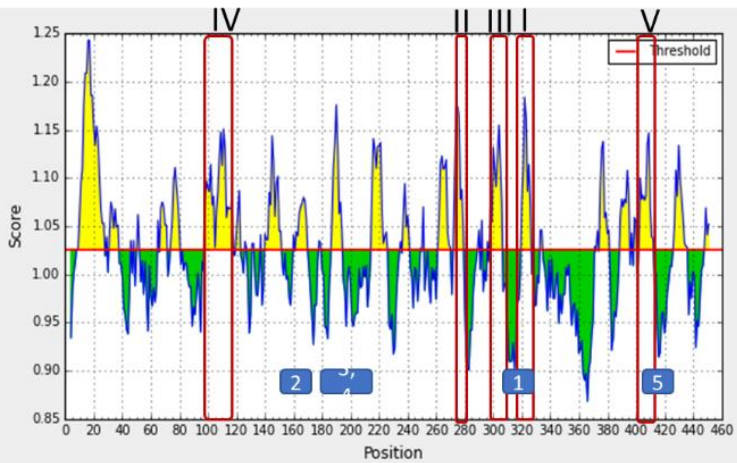

C

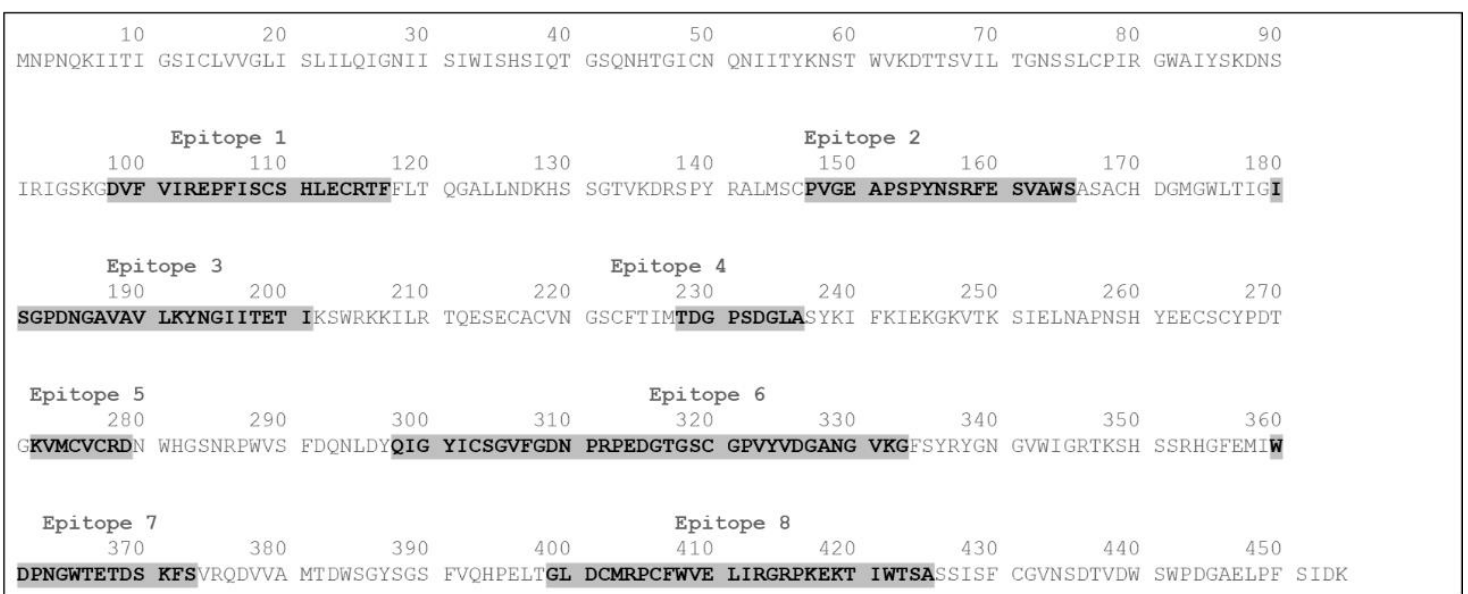

Figure 1: Epitopes prediction for the neuraminidase protein from influenza A/Puerto Rico /8/1934 (H1N1) virus. Panel A - Bepipred linear epitope prediction method. Panel B - Kolaskar \& Tongaonkar antigenic method. Numbers I-V indicate top five epitopes according to amino acid score belonging the sequence in both methods. The numbering 1-5 (bottom squares) indicate top 5 peptides epitopes predicted by CTLPred method. Panel C: Linear sequence of amino acid residues of neuraminidase protein from influenza A/Puerto Rico/8/1934 virus strain (GenBank: ABO21711.1). The eight putative epitopes after aligning of 15 epitopes originated by three different prediction methods are showed in bold and highlighted.

Source: Authors' data

The 7,438 sequences of the neuraminidase (NA) protein of influenza A (H1N1) virus obtained from bioproject databank are from 2009-2017, after the influenza A pandemic 2009 outbreak. All sequences were aligned with the presumed 8 epitopes obtained by the computational methods. Several amino acid residues substitutions in the NA protein sequence could be noticed within the epitopes, but they were discharged for further analysis. Modifications that had low amino acid residue replacement were unconsidered if their prevalence was less than $30 / 7,438(<0.4 \%)$ in the protein sequences, indicating low prevalence in the viral population; suggesting it was eliminated by the vaccination program and/or it was not suitable to remain in the viral population. On the other hand, the number of sequences containing amino acid residue substitutions higher than 30 (>0.4\%) was considered relevant 
for further analysis; indicating likely modification, survival in the viral population or immune response escape. The results showed the presence of 21 relevant substitution points correlated to the eight epitopes obtained (FIG. 2A). Besides, others non-relevant substitution points were observed and discharged (frequency $<0.4 \%$ ). The stability of each substitution point in the virus population from 2009-2017 was verified by dividing the number of amino acid substitutions counted by the total number of protein sequences, yearly and four substitutions were observed (FIG. 2B) and remain substitutions (17) occurred before 2009 (not shown).

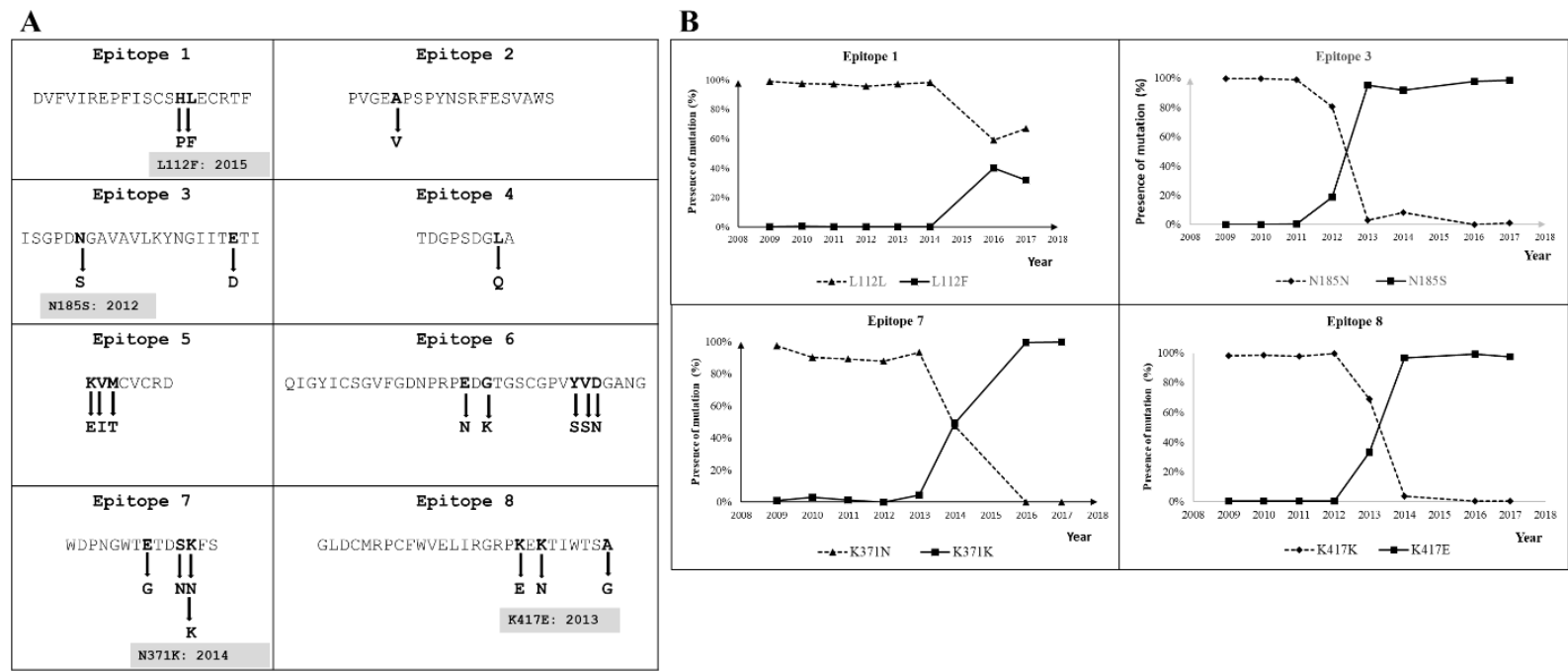

Figure 2: Presence of amino acid substitutions in the neuraminidase of influenza virus A (H1N1) population from 2009 to 2017 related to the 8 analyzed epitopes Panel A: Major amino acid substitution points noticed in the neuraminidase sequences compared to selected epitopes. The eight selected epitopes were aligned to the 7,438 neuraminidase protein sequences and the main amino acid substitution points are showed by bold letters. Four relevant amino acid substitution were observed from 2009 to 2017 indicated by amino acid substitution in the year and the others amino acid substitution points had occurred before 2009. Panel B: Amino acids residues have been replaced in the epitopes 1, 3, 7 and 8 in the considered period, which could be impacted the viral population against the used vaccine.

Source: Authors' data

It was noticed that $81.0 \%(17 / 21)$ of the amino acid substitution points remained stable in the viral population from 2009-2017 (H111P, A151V, E199D, D233N, L235Q, K272E, V273I, M274T, E314N, G316K, Y324S, V325S, D326N, E367G, S370N, K419N, and A425G) compared to the NA protein from influenza A/PuertoRico/8/1934 (H1N1) virus. Another 19\% (4/21) of the amino acid substitution points had occurred during the analysis period (L112F, N185S, N371K, and K417E), thereby changing the viral population profile (FIG. 2).

The amino acid substitution I196V (Epitope 3) and M274I (Epitope 5) showed population peaks of $1.5 \%$ and $6.3 \%$, respectively in 2012, and the amino acid substitution 
T317K (Epitope 6) with 8\% peak in 2010 (Not shown). However, the amino acid substitution K272T (Epitope 5) was observed in two NA sequences (ABO38068-A/Brazil/11/1978 and AFO65052-A/Brazil/74200/2000) in the period previously than the amino acid substitution K272E; suggesting a modification of viral profile amino acid substitution from KVM (1934) to TVM (before 2009), and then it replaced completely to EIT (2009), which remained in the viral population until 2017 (FIG. 2). Additionally, the amino acid substitution V325F (Epitope 6) was noticed in $0.53 \%$ of the NA protein sequences in 2009 , but the amino acid substitution V325S remained in the viral population (FIG. 2). In the epitope 1, the amino acid substitution L112F was noticed in 40\% and 32\% of NA proteins in $2016(\mathrm{n}=132)$ and $2017(\mathrm{n}=420)$, respectively (FIG. 2), indicating the necessity to monitor this epitope in the coming years. Besides, the amino acid substitutions noticed in the epitopes 2, 4, 5, and 7 remained stable in the viral population in the years 2009-2017 (FIG. 2A).

Also, the NA protein sequence from influenza A/PuertoRico/8/1934 (H1N1) virus submitted to Phyre 2 protein modelling had $100 \%$ of confidence of the model. ${ }^{20}$ This way, the amino acid substitutions found in the epitopes 1, 3, 7 e 8 (FIG. 2) were checked for their positions in the NA protein and only one (A425G) was found to be inside the molecule (FIG. $3)$.
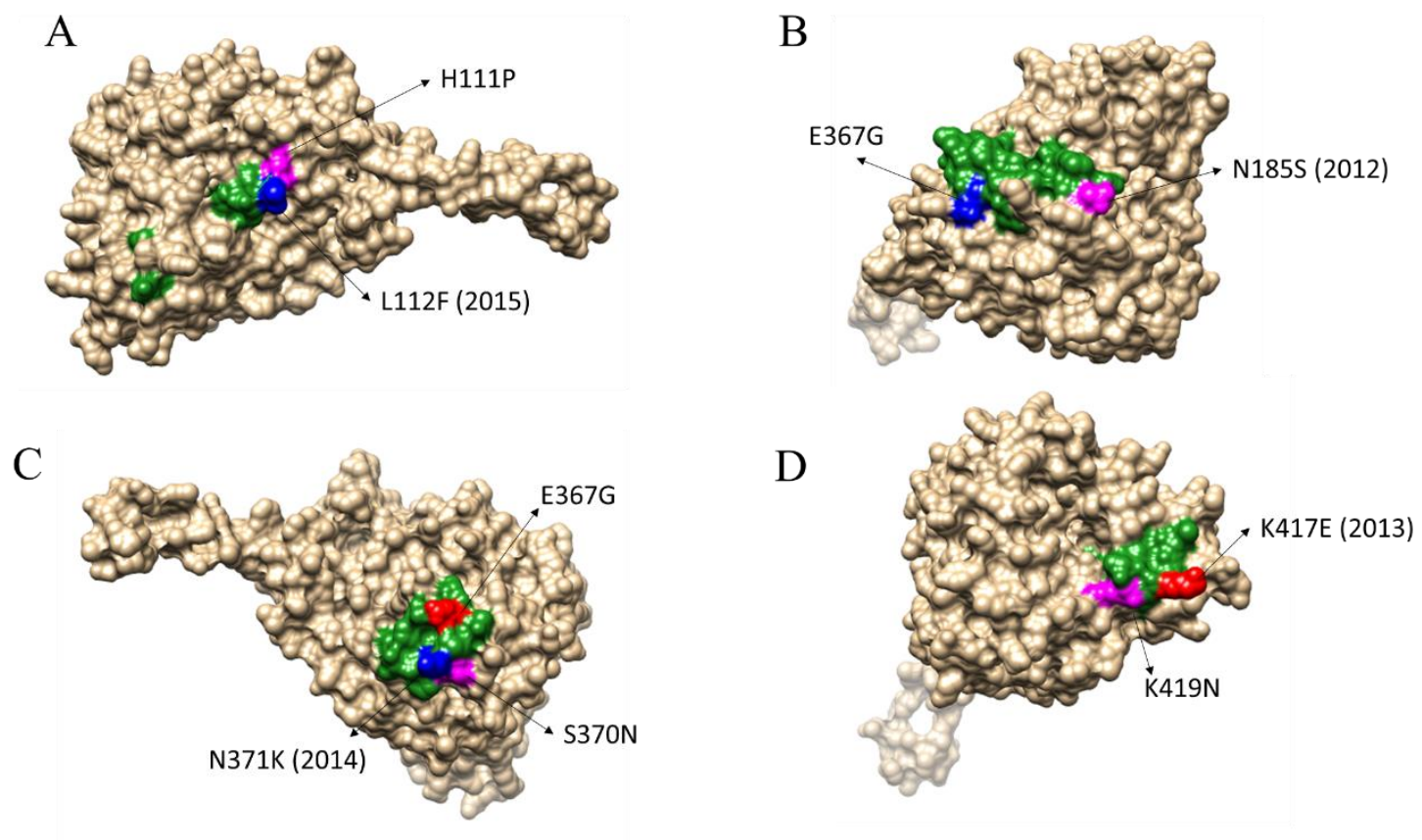
Figure 3: Position of the amino acid substitutions in the neuraminidase of influenza virus A (H1N1) strain Puerto Rico (1934) in the period of 2009-2017. Panel A: Epitope 1 (Green) containing amino acid substitution H111P (magenta), and L112F (Blue). Panel B: Epitope 3 (Green) containing amino acid substitution N185S (magenta), and E199D (Blue). Panel C: Epitope 7 (Green) containing amino acid substitution E367G (Red), S370N (magenta), and N371K (Blue). Panel D: Epitope 8 (Green) containing amino acid substitution K417E (Red), K419N (magenta), and $\mathrm{A} 425 \mathrm{G}$ hidden amino acid residue (Blue, not visible).

Source: Authors' data

The vaccine that was used in Brazil to protect the population against $\mathrm{H} 1 \mathrm{~N} 1$ influenza virus $\mathrm{A}$ in 2017 was the influenza virus subtype A/California/7/2009 and the World Health Organization recommended that it should be replaced with A/Michigan/45/2015 virus subtype in the vaccine for 2018. ${ }^{18,19}$ Thus, the suggested epitopes 1 through 8 were modified to contain the amino acids present in the 2017 viral population according to the results obtained here (FIG. 2) and aligned with the neuraminidase of the H1N1 A/California/7/2009 and A/Michigan/45/2015 virus subtypes (FIG. 4). The known deleted 15 amino acids sequence (YVNISNTNFAAGQSV) in the reference sequence A/Puerto Rico/1934 was observed during sequence alignment with the 8 epitopes (FIG. 4), but this reference sequence was used as the basis for the amino acids residues numbering. This deleted sequence could be a sequencing error in the influenza virus subtype A/Puerto Rico/1934 or a recombination virus acquiring this sequence through the years. ${ }^{5}$ 
Epitopes1-8modif . A/California/07/2009 A/Michigan/45/2015

Epitopes1-8modif . A/California/07/2009 A/Michigan/45/2015

Epitopes1-8modif . A/California/07/2009 A/Michigan/45/2015

Epitopes1-8modif. A/California/07/2009 A/Michigan/45/2015

Epitopes1-8modif. A/California/07/2009 A/Michigan/45/2015

Epitopes1-8modif . A/California/07/2009 A/Michigan/45/2015

Epitopes1-8modif . A/California/07/2009 A/Michigan/45/2015

Epitopes1-8modif. A/California/07/2009 A/Michigan/45/2015
MNPNQKIITIGSVCMTIGMANLILQI GNI ISIWISHS IQLGNQNQIETCNQSVITYENNT MNPNQKIITIGS I CMTI GMANL I LQI GN I IS I WVS HS IQ I GNQSQIETCNQSV ITYENNT

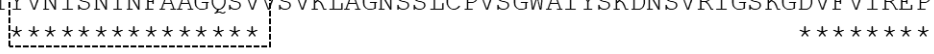

FISCSP FERTE-------------------------PIGEVPS PYNSRFESVAWS FISCSP LECRTFFLTQGALLNDKHSNGT IKDRSPYRT LMSCP I GEVPS PYNSRFESVAWS FISCSP LECRTFFLTQGALLNDKHSNGT IKDRSPYRTLMSCP IGEVPS PYNSRFESVAWS

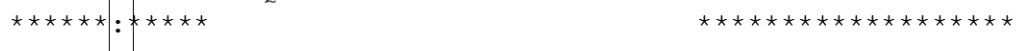

------------ISGPDSGAVAVLKYNGIITDTI--------------------ASACHDGINWLTI GI SGPDNGAVAVLKYNGI I TDT IKSWRNN I LRTQESECACVNGSCFT ASACHDGINWLTI GI SGPDSGAVAVLKYNGI I TDT IKSWRNN I LRTQE SECACVNGSCFT

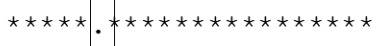

--TDGPSDGQA----------------------------EITCVCRD----VMTDGP SNGQASYKI FRIEKGKIVKSVEMNAPNYHYEECSCYPDSSEITCVCRDNWHGSN IMTDGP SDGQASYKI FRIEKGKI I KSVEMKAPNYHYEECSCYPDSSEITCVCRDNWHGSN $* \star * * *: \forall * * \quad: \quad: \quad * * * \star \star \star * *$

----------QIGYICS GV $\$$ GDNPRPNDKT GSCGPVSSNGANGVKG---------RPWVSENQNLEYQIGYICS IIFGDNPRPNDKTGSCGPVSSNGANGVKGFS FKYGNGVWIG RPWVSENQNLEYQMGYICSGV $F$ GNPRPNDKTGSCGPVSSNGANGVKGFSFKYGNGVWIG

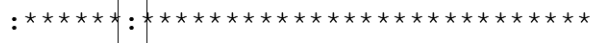
-WDPNGWTGTDNKES GLDCIRP RTKS ISSRNGFEMIWDPNGWTGTDNNES IKQDIVGINEWSGYSGSEVQHPELTGLDCIRP RTKS ISSRKGFEMIWDPNGWTGTDNKFS IKQDIVGINEWSGYSGSFVQHPELTGLDCIRP

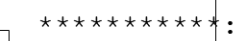

CEWVELIRGRPEENTIWTSG----------------------

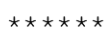

CEWVELIRGRPKENTIWTSGSS ISECGVNSDTVGWSWPDGAELPFTIDK CFWVELIRGRPEENTIWTSGSS ISECGVNSDTVGWSWPDGAELPFTIDK

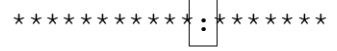

Figure 4: Clustal omega alignment of epitopes 1-8 modified to fit the four amino acid substitutions in NA protein detected in this study (lined squares) against NA protein from influenza A/California/7/2009 and A/Michigan/45/2015 virus subtypes. The 15 amino acid residues deleted in the NA protein sequence of influenza A/Puerto Rico/8/1934 subtype virus are showed (hatched square).

Source: Authors' data

The alignment showed that epitopes 2,5 , and 6 had no modifications in the two subtype virus sequences (FIG. 4). The subtype virus A/Michigan/45/2105 had the amino acid substitution N185S (epitope 3) and maintained the amino acid residue aspartate (D) at position 233 (epitope 4), while the subtype A/California/7/2009 had no amino acid substitution at position 185 (epitope 3) but replaced the amino acid residue at position 233 (D233N) in the epitope 4. Thus, the new component of the vaccine could favor the immunological response of the vaccine to protect against the supposed $\mathrm{H} 1 \mathrm{~N} 1$ viral population, which has been circulating 
in Brazil from 2018 and avoiding an outbreak. Another observed amino acid substitution in the viral population is in epitope 7 (N371K) from 2014 (FIG. 2), which was present in the virus subtype A/Michigan/45/2015 (FIG. 3). In conclusion, the influenza virus subtype A/Michigan/45/2015 is closer to the newer subtype H1N1 viral population, which was supposedly circulating in Brazil in 2018.

The amino acid substitution K417E present in the epitope 8 and noticed in the viral population from 2013 had the tendency to remain in the viral population (FIG. 2). This amino acid substitution was not detected in the influenza virus A/California/7/2009, but it was present in the A/Michigan/45/2015 (FIG. 3). Besides these analyzed modifications, the influenza virus A/Michigan/45/2015 presents four different amino acid residues compared to the virus A/California/7/2009, which was not considered in the putative epitopes (FIG. 4).

The amino acid substitution L112F present in the putative epitope 1 was noticed in $40 \%$ and 32\% of the viral neuraminidase sequences in $2016(n=132)$ and $2017(n=420)$, respectively (FIG. 2). Neither the influenza virus subtypes A/California/7/2009 nor A/Michigan/45/2015 presents this amino acid substitution in this position (FIG. 4). In addition, the free energy of the NA protein was analyzed using STRUMM ${ }^{21}$ had predicted that L112F could have energy of 0.06 ddGs more stable than L112L and it is considered to be outside the NA 3D molecule (FIG.3).

\section{DISCUSSION}

In this study, we presented an in silico analysis investigating the impact of the amino acid substitutions in putative epitopes on the vaccine strains of type A influenza virus H1N1 and in the viral population from 2009-2017. The Bepipred method predicts linear epitopes of B cells combining two methodologies. ${ }^{22}$ This method uses HMM (Hidden Markov Model) with the propensity scale previously suggested by Parker et al. (1986). ${ }^{23}$ The Kolaskar \& Tongaonkar method $^{24}$ is a tool of the IEDB (Immune Epitope Database), which predicts B cell epitopes using the physico-chemical properties of the molecules and amino acid residues as reference. It also considers their frequencies in the experimental epitopes through semi-empirical methods and it has an efficacy of $75 \% .{ }^{25}$ In contrast, the CTL Pred (Cytotoxic T-Lymphocyte Prediction) method is based on quantitative matrix using support vector machine and artificial neural networks, following a standard organization, where it executes and tests the epitope of $\mathrm{T}$ cells in its database. ${ }^{26}$ Each of these methods has its own particularities, the Bepipred and Kolaskar 
\& Tongaonkar methods share similar predictions based on B cell epitope compared to the CTL Pred that uses cytotoxic T cells. Additionally, the Kolaskar \& Tongaonkar method is more precise than Bepipred since it uses experimental sequence available in its database. ${ }^{26}$ The CTL Pred is a direct method and it is utilized for epitope prediction. ${ }^{27}$

Our results showed 8 suggested epitopes (FIG. 1), of which epitope 3 was predicted by all three methods, while epitopes 1 and 4 were suggested only by Kolaskar \& Tongaonkar and Bepipred methods, respectively. The identification and selection of the main epitopes in silico and/or by experimental data is an important stage during the development of vaccines, ${ }^{6}$ as well as immunotherapies and/or diagnostics.

The amino acid substitutions observed in epitopes 1, 3, 7, and 8 from 2009-2017 (FIG. 2 and FIG. 3) for the 7,438 NA sequences globally suggest that the viral population has mutated due to vaccination and environmental pressures, yearly, since at least one amino acid substitution per year was observed (FIG. 3). Additionally, the same pattern of amino acid substitution in H1N1 influenza virus was noticed in 140 NA sequences sampled from Rio Grande do Sul (Brazil), indicating that the amino acid substitution is not geographical but time dependent, since the Brazilian cases of H1N1 showed conserved virus among them and they shared the same characteristics with the circulating virus from others regions worldwide, and it is in accordance with the findings of Popova et al. (2019). ${ }^{5}$ According to figure 2 , four amino acid substitutions (19.0\%) changed the NA sequence of viral population in this period (N185S in 2012, K417E in 2013, N371K in 2014, and L112F in 2015), and the remaining 76.2\% amino acid substitutions (16/21), which occurred before 2009, have persisted in this population (H111P, A151V, E199D, L235Q, K272E, V273I, M274T, E314N, G316K, Y324S, V325S, D326N, E367G, S370N, K419N and A425G). Furthermore, 4.8\% (1/21) amino acid substitution (D233N) returned to the original amino acid residue (D) before 2009 $(\mathrm{D} 233 \mathrm{~N} \rightarrow \mathrm{N} 233 \mathrm{D})$ compared to the NA protein from influenza A/PuertoRico/8/1934 (H1N1) virus (FIG. 2). Next, if these observed amino acid substitutions markedly changed the neuraminidase sequence and also the amino acid substitutions in the other proteins of the virus (not analyzed in the current study), then it could result in a new virus strain subtype, which could escape from antibodies induced by the current vaccine. The four amino acid substitutions and others observed here are in outside of the NA protein, indicating that epitopes are necessary for 3D structural conformation of the molecule (FIG. 3). Therefore, the H1N1 virus and the new H1N1 variants should be closely monitored to check the circulating virus and their 
characteristics such as virulence, transmission and/or antiviral resistance, and the efficacy of the current vaccine. ${ }^{28}$

The amino acid substitution L112F present in epitope 1 was noticed in $40 \%$ and $32 \%$ of the viral neuraminidase sequences in $2016(n=132)$ and $2017(n=420)$, respectively (FIG. 2) and needs be closely monitored in the future. Both the influenza virus subtypes A/California/7/2009 (former Brazilian vaccine) and A/Michigan/45/2015 (Current Brazilian vaccine) did not present this amino acid modification at this position. The replacement of Leucine (L) by Phenylalanine (F) could increase the stability of the NA protein or virulence of the virus by 0.06 ddGs using the STRUM analysis. ${ }^{21}$ Next, if the critical amino acids in an epitope are replaced by another, this could change the conformation of the epitope and also its ligation to the antibodies. ${ }^{29,30}$ Therefore, the identification of the amino acid substitution L112F in epitope 1 is critical for the epitope and also for peptides or proteins designs related to vaccine or antibodies productions should be done. ${ }^{31}$ The amino acid substitutions observed during the years 2012-2015 in the surface glycoprotein NA could be responsible for the inability of the host to develop a permanent resistance against the H1N1 virus. This could initiate a new influenza pandemic and require a change of the vaccine H1N1 strain. 5,7,32,33

Corroborating the World Health Organization, the circulation of distinct strains of influenza A (H1N1) was demonstrated from 2009-2017 and all epitopes for NA were closely related to the prototype vaccine strain A/Michigan/45/2015, which was incorporated in the Brazilian vaccine. ${ }^{19}$ Since the strain A/Michigan/45/2015 did not harbor the amino acid substitution L112F detected in the circulating viral population in 2016 and 2017, and probably in 2018, we cannot preview the impact and consequences of the immunization process in the selection of this new variant of H1N1 influenza virus. Thus, the amino acid substitution L112F should be closely monitored in 2018 and beyond to assess if it remains in the viral population, and also for the protection of vaccine efficacy.

\section{CONCLUSION}

The findings of this study indicate that the replacement of influenza virus A (H1N1) subtype in the Brazilian vaccine is necessary, and it corroborates the recommendation of the World Health Organization related to the surface glycoprotein neuraminidase as well as the importance of bioinformatics data analysis. 


\section{ACKNOWLEDGMENTS}

We thank The Foundation for Research of the State of Minas Gerais (FAPEMIG).

\section{REFERENCES}

1 CHAMBERS, Benjamin S. et al. Recent H3N2 influenza virus clinical isolates rapidly acquire hemagglutinin or neuraminidase mutations when propagated for antigenic analyses. Journal of Virology, v. 88, n. 18, p. 10986-10989, 2014.

2 PARADIS, Eric G. et al. Impact of the H275Y and I223V mutations in the neuraminidase of the 2009 pandemic influenza virus in vitro and evaluating experimental reproducibility. PLoS One, v. 10, n. 5; p. e0126115, 2015.

3 SHAO, Wenhan et al. Evolution of influenza a virus by mutation and re-assortment. International journal of molecular sciences, v. 18, n. 8, p. 1650-1662, 2017.

4 WU, Shuangsheng et al. Mortality burden from seasonal influenza and 2009 H1N1 pandemic influenza in Beijing, China, 2007-2013. Influenza and Other Respiratory Viruses, v. 12, n. 1, p. 88-97, 2018.

5 POPOVA, Anfisa V. et al. Allele-specific nonstationarity in evolution of influenza A virus surface proteins. Proceedings of the National Academy of Sciences, v. 116, n.42, p. 21104-21112, 2019.

6 ANDERSON, Christopher S. et al. Antigenic cartography of H1N1 influenza viruses using sequence-based antigenic distance calculation. BMC Bioinformatics, v. 19, n. 1, p. 51-61, 2018.

7 PETROVA, Velislava N.; RUSSELL, Colin A. The evolution of seasonal influenza viruses. Nature Review Microbiolology, v. 16, n. 1, p. 47-60, 2018.

8 BUSH, Robin M. Influenza Evolution. In: Encyclopedia of infectious diseases: Modern Methodologies. J Wiley \& Sons, New York, USA, p. 199-214, 2007.

9 GOTTSCHALK, Alfred. Neuraminidase: the specific enzyme of influenza virus and Vibrio cholerae. BBA - Biochimica et Biophysica Acta, v. 23, n. C, p. 645-646, 1957.

10 ABED, Yacine et al. Impact of potential permissive neuraminidase mutations on viral fitness of the H275Y Oseltamivir-resistant Influenza A(H1N1)pdm09 virus in vitro, in mice and in ferrets. Journal of Virology, v. 88, n. 3, p. 1652-1658, 2014. 
11 MOSCONA, Anne. Neuraminidase inhibitors for influenza. The New England Journal of Medicine, v. 353, n. 13, p. 1363-1373, 2005.

12 SUZUKI, Takashi et al. Sialidase activity of influenza A virus in an endocytic pathway enhances viral replication. Journal of Virology, v. 79, n.18, p. 11705-11715, 2005.

13 MATROSOVICH, Mikhail N. et al. Neuraminidase is important for the initiation of influenza virus infection in human airway epithelium. Journal of Virology, v. 78, n. 22, p. 12665-12667, 2004.

14 ABED, Yacine; BAZ, Mariana; BOIVIN, Guy. Impact of neuraminidase mutations conferring influenza resistance to neuraminidase inhibitors in the $\mathrm{N} 1$ and $\mathrm{N} 2$ genetic backgrounds. Antiviral Therapy, v. 11, n. 8, p. 971-976, 2006.

15 AOKI, Fred Y.; BOIVIN, Guy; ROBERTS, Noel. Influenza virus susceptibility and resistance to Oseltamivir. Antiviral Therapy, v. 12, n. 4 Pt B, p. 603-616, 2007.

16 BUTLER, Jeff et al. Estimating the fitness advantage conferred by permissive neuraminidase mutations in recent Oseltamivir-resistant A(H1N1)pdm09 Influenza Viruses. PLoS Pathogens, v. 10, n. 4, p. e1004065, 2014.

17 PETRIE, Joshua G. et al. Antibodies against the current influenza a (H1N1) vaccine strain do not protect some individuals from infection with contemporary circulating influenza A (H1N1) virus strains. Journal of Infectious Diseases, v. 214, n. 12, p. 19471951, 2016.

18 WORLD HEALTH ORGANIZATION - WHO. Recommended composition of influenza virus vaccines for use in the 2017-2018 northern hemisphere influenza season. Weekly Epidemiologycal Record, v. 92, n. 11, p. 117-128, 2017.

19 BRASIL. Ministério da Saúde. Resolução - RE No 2.696, de 6 de outubro de 2017. Aprova as vacinas influenza a serem comercializadas ou utilizadas no Brasil no ano de 2018. Diário Oficial da República Federativa do Brasil, v. 194, p. 76, 2017.

20 KELLEY, Lawrence A. et al. The Phyre2 web portal for protein modeling, prediction and analysis. Nature Protocols, v. 10, n. 6, p. 845-858, 2015.

21 QUAN, Lijun; LV, Qiang; ZHANG, Yang. STRUM: Structure-based prediction of protein stability changes upon single-point mutation. Bioinformatics, v. 32, n. 19, p. 2936-2946, 2016.

22 LARSEN, Jens E.P.; LUND, Ole; NIELSEN, Morten. Improved method for predicting linear B-cell epitopes. Immunome Research, v. 2, n. 1, p. 2, 2006.

23 PARKER, J. M. R.; GUO, D.; HODGES, R. S. New hydrophilicity scale derived from 
high-performance liquid chromatography peptide retention data: correlation of predicted surface residues with antigenicity and $\mathrm{x}$-ray-derived accessible sites. Biochemistry, v. 25, n. 19, p. 5425-5432, 1986.

24 KOLASKAR, Ashok S.; TONGAONKAR, Prasad C. A semi-empirical method for prediction of antigenic determinants on protein antigens. FEBS Letters, v. 276, n. 1, p. 172-174, 1990.

25 VITA, Randi et al. The Immune Epitope Database 2.0. Nucleic Acids Research, v. 38, n. suppl. 1, p. D854-D862, 2009.

26 BHASIN, Manoy; RAGHAVA, Gajendra P.S. Prediction of CTL epitopes using QM, SVM and ANN techniques. Vaccine, v. 22, n. 23, p. 3195-3204, 2004.

27 FOGED, Camilla; HANSEN, Jon; AGGER, Else M. License to kill: Formulation requirements for optimal priming of CD8 + CTL responses with particulate vaccine delivery systems. European Journal of Pharmaceutical Sciences, v. 45, n. 4, p. $482-$ 491, 2012.

28 MELIDOU, Angeliki et al. Molecular and phylogenetic analysis of the haemagglutinin gene of pandemic influenza H1N1 2009 viruses associated with severe and fatal infections. Virus Research, v. 151, n. 2, p. 192-199, 2010.

29 ANDERSEN, Pernille H.; NIELSEN, Morten; LUND, Ole. Prediction of residues in discontinuous B-cell epitopes using protein 3D structures. Protein Science, v. 15, n. 11, p. 2558-2567, 2006.

30 WANG, Yulong et al. Determinants of antigenicity and specificity in immune response for protein sequences. BMC Bioinformatics, v. 12, n. 1, p. 251-263, 2011.

31 TRIER, N. H.; HANSEN, P. R.; HOUEN, G. 2012. Production and characterization of peptide antibodies. Methods, v. 56, n. 2, p. 136-144, 2012.

32 COX, N. J.; SUBBARAO, K. 2000. Global epidemiology of influenza: past and present. Annual Review of Medicine, v. 51, n. 1, p. 407-21, 2000.

33 SMITH, Derek J. et al. Mapping the antigenic and genetic evolution of influenza virus. Science, v. 305, n. 5682, p. 371-376, 2004. 